CERN-PPE/95-52

18 April 1995

\title{
SUMMARY OF LOW-MASS DILEPTON AND DIRECT PHOTON RESULTS
}

\author{
Itzhak Tserruya * \\ Department of Particle Physics, Weizmann Institute, \\ Rehovot 76100, Israel \\ and \\ PPE Division, CERN, \\ CH-1211 Geneva, Switzerland
}

\begin{abstract}
An enhanced production of dileptons $-\mathrm{e}^{+} \mathrm{e}^{-}$or $\mu^{+} \mu^{-}$pairs -, over those expected from known hadronic sources, has been observed by CERES, HELIOS-3 and NA38 in S-induced interactions on heavy targets at $200 \mathrm{GeV} /$ nucleon. The excess covers the mass range from $200 \mathrm{MeV} / \mathrm{c}^{2}$ up to the $J / \psi$. There is no clear evidence of a signal in the search for direct photons. All the experimental results seem to be fairly consistent.
\end{abstract}

Plenary Talk at Quark Matter '95, Monterey/California, January 9 - 13, 1995.

*Work supported by the MINERVA Foundation, Munich, Germany and the H. Gutwirth Fund for the promotion of research at the Weizmann Institute. 


\section{INTRODUCTION}

There is a clear and interesting message emerging from the three experiments measuring lepton pairs, HELIOS-3, NA38 and NA45/CERES; they have all recently reported results on S-induced interactions showing an enhanced production of dileptons - either $\mathrm{e}^{+} \mathrm{e}^{-}$or $\mu^{+} \mu^{-}$pairs -, over a very broad invariant-mass range, from $\mathrm{m}_{l l} \sim 200 \mathrm{MeV} / \mathrm{c}^{2}$ up to the $J / \psi$. I was asked to summarize these results on dileptons and also those on direct photons, which in principle should carry the same physics information, and in particular to assess their consistency. In the following I explicitly refer to dileptons (virtual photons) but most of the arguments apply as well to real photons.

The measurement of dileptons has always been emphasized as one of the most relevant probes to study the dynamics of relativistic heavy-ion collisions. The argument is simple and was first proposed by Shuryak more than sixteen years ago [1]: since dileptons interact only electromagnetically, their mean free path is large compared to the size of the system formed in these collisions; therefore once produced, they can leave the interaction region and reach the detectors without any further interaction, carrying information about the conditions and properties of the matter at the time of their production.

Dileptons can be emitted throughout the entire collision. Various processes are involved, and to the extent that they have distinct characteristics, a careful analysis should be able, at least in principle, to disentangle the whole space-time evolution of the collision. I will follow the collision as a function of time to briefly discuss the various contributions. The first one comes from the primary hard collisions which drive the system towards equilibrium. Dileptons are directly produced there by the Drell-Yan process of partonantiparton annihilation into $l^{+} l^{-}$. Heavy flavor mesons $(D \bar{D})$ also produced in these primary collisions lead at a later stage to uncorrelated pairs from their semi-leptonic decay. These are the dominant contributions to the dilepton continuum spectrum in hadron-hadron collisions at masses $\mathrm{m}>1.5 \mathrm{GeV} / \mathrm{c}^{2}$.

Once the system reaches thermal equilibrium, dileptons are emitted as thermal radiation during the expansion and cooling phases up to the freeze-out time when the hadrons cease to interact. The identification of the thermal radiation is the main topic of interest. It could tell us about the nature of the matter formed, the conjectured quark-gluon plasma (QGP) or a high-density hadron gas (HG). The relative strength of the elementary processes involved $-q \bar{q}$ annihilation in the QGP phase and $\pi^{+} \pi^{-}$annihilation in the HG phase- is expected to be different. The thermal radiation emission rate is a strongly increasing function of the temperature; it is therefore most abundantly emitted at the early stages when the temperature and the energy density have their largest values, thereby providing a higher sensitivity to identify the thermal radiation from the QGP, if it is formed. This sensitivity increases as the initial temperature of the system increases relatively to the critical temperature of the phase transition. Yet, the absolute yields, obtained by integrating the emission rate over the space-time evolution of the collision, are theoretically uncertain as the calculations critically depend on the parameters of the time evolution.

The spectral shape of the thermal radiation from partons and hadrons is also expected to be different. The $\pi^{+} \pi^{-}$annihilation cross section is dominated by the pole at the $\rho$ mass of the pion electromagnetic form factor and it obviously has a threshold at $2 \mathrm{~m}_{\pi}$. 
The window to search for the thermal radiation from the HG is at low masses, around and below the $\rho$-meson mass [2-4]. On the other hand, the $q \bar{q}$ annihilation produces an essentially exponential spectrum with a slope parameter reflecting the temperature of the system; the threshold, given by $2 \mathrm{~m}_{q}$, is expected to be negligible [5]. Theoretical calculations have singled out the mass range of $1-3 \mathrm{GeV} / \mathrm{c}^{2}$ as the most appropriate window to observe the thermal radiation from the QGP phase $[2,6]$.

A large amount of additional lepton pairs is produced at a late stage, long after the freeze-out time, by the electromagnetic decay of hadrons $\left(\pi^{\circ}, \eta, \eta \rightarrow \mathrm{e}^{+} \mathrm{e}^{-} \gamma, \omega \rightarrow \mathrm{e}^{+} \mathrm{e}^{-} \pi^{\circ}\right.$, $\left.\omega, \phi \rightarrow \mathrm{e}^{+} \mathrm{e}^{-}\right)$. It is the main source of dileptons at masses $\mathrm{m} \leq 1 \mathrm{GeV} / \mathrm{c}^{2}$ in hadron-hadron collisions.

In addition to all these sources, bremstrahlung emission of soft virtual photons takes place both in the QGP and in HG phases. They mainly contribute to the very low masses of dielectrons $[3,4,7]$.

The vector mesons $\rho, \omega$ and $\phi$, measured through their $l^{+} l^{-}$-decay channels, are also considered as important messengers of the collision dynamics. The $\rho$-meson, in particular, has been proposed as a potential signal of the restoration of chiral symmetry which is expected to occur at conditions similar to those leading to deconfinement [8,9]. Its mass and width are expected to be strongly modified as the system approaches chiral restoration. Due to its very short lifetime of $1.3 \mathrm{fm} / \mathrm{c}$, the decay $\rho \rightarrow l^{+} l^{-}$will occur inside the dense matter and the dilepton mass spectrum may indicate such a transition. The short lifetime offers also another attractive possibility; several generations of $\rho$-mesons could be produced during the lifetime of the system [10], as a result of which the $\rho$-yield would increase, relative to the $\omega$, and could serve as a clock to measure the fireball lifetime.

The interest in the $\phi$-meson is quite different. It has a lifetime of $\tau=48 \mathrm{fm} / \mathrm{c}$, sufficiently large to decay outside the interaction volume. Consisting of $s \bar{s}$ quarks, it has been proposed as a probe of QGP formation [11]. Its production is predicted to be enhanced as an abundant production of $s \bar{s}$ is expected if chemical equilibrium is achieved in the plasma; furthermore the OZI rule which suppresses $\phi$ formation in hadron-hadron collisions should become irrelevant in the case of QGP formation.

After discussing the interest in electromagnetic probes, it is important to appreciate the difficulty of the measurements, which explains why the first results became available only recently. The main problem in the case of dileptons is the huge combinatorial background of uncorrelated pairs originating from the decay of hadronic particles (and also from conversions in the measurements of electron pairs), which strongly increases as the coverage moves to the low-mass and low- $\mathrm{p}_{t}$ regions. This is one of the factors limiting the sensitivity to any new source. Another limit is set by the uncertainties in the contributions from the known sources, i.e. the physics background; the Drell-Yan and open charm contributions (at masses $\mathrm{m}>1.5 \mathrm{GeV} / \mathrm{c}^{2}$ ) and the hadronic sources (at masses $\mathrm{m}<1$ $\left.\mathrm{GeV} / \mathrm{c}^{2}\right)$ must be well under control before a claim can be made on the observation of a new source. Under these circumstances, the best approach is a systematic one, performing precise measurements of the dilepton production in $\mathrm{pp}$ and $\mathrm{pA}$ collisions as the basis for identifying any possible deviation from the known physics in nucleus-nucleus collisions.

The first results which I will review below show clear evidences of such deviations.

The paper is organized as follows. Section 2 briefly presents all the experiments involved in the measurement of electromagnetic observables in ultra-relativistic heavy-ion collisions 
and their main results. In Section 3, a quantitative comparison is attempted in order to evaluate the consistency among the different experimental results. Section 4 contains a short discussion on open questions, possible interpretations and further work.

\section{EXPERIMENTAL RESULTS ON ELECTROMAGNETIC PROBES}

The experiments involved in the measurement of electromagnetic probes are listed in Table 1. Two are dedicated to the measurement of muon pairs, HELIOS-3 which covers

Table 1

List of measurements on electromagnetic probes

\begin{tabular}{|c|c|c|c|c|}
\hline Probe & Experiment & System & $y$ & Range (GeV) \\
\hline $\begin{array}{cc} & \mu^{+} \mu^{-} \\
\text {Dileptons } & \mu^{+} \mu^{-} \\
& e^{+} e^{-}\end{array}$ & $\begin{array}{l}\text { HELIOS-3 } \\
\text { NA38 } \\
\text { CERES }\end{array}$ & $\begin{array}{cc}p-W & S-W \\
p-W & S-U \\
p-B e, A u & S-A u\end{array}$ & $\begin{array}{l}3.5-5.5 \\
3.0-4.0 \\
2.1-2.7\end{array}$ & $\begin{aligned} m & =0.3-4.0 \\
m & \sim 0.6-4.0 \\
m & =0.2-1.4\end{aligned}$ \\
\hline Photons & $\begin{array}{l}\text { CERES } \\
\text { WA } 80 \\
\text { HELIOS-2 }\end{array}$ & $\begin{array}{c}S-A u \\
S-A u \\
p, O, S-W, P t\end{array}$ & $\begin{array}{l}2.1-2.7 \\
2.1-2.9 \\
1.0-1.9\end{array}$ & $\begin{array}{l}p_{t}=0.4-2.0 \\
p_{t}=0.5-3.0 \\
p_{t}=0.1-1.5\end{array}$ \\
\hline
\end{tabular}

a very broad mass range from the dimuon threshold up to the $J / \psi$, and NA38 which is mostly focused on the mass range around and below the $J / \psi$. The acceptances of the two muon experiments are listed in the Table. CERES/NA45 is the only experiment dedicated to the measurement of low-mass electron pairs, from $\sim 50 \mathrm{MeV} / \mathrm{c}^{2}$ up to beyond $1 \mathrm{GeV} / \mathrm{c}^{2}$. It covers the mid-rapidity region with a very broad range of $p_{t}$. The reference measurements on $\mathrm{pp}$ and $\mathrm{pA}$ collisions performed by each experiment are also listed in Table 1. Two experiments are presently involved in the measurement of direct photons; WA80 which measures them directly and CERES which uses the conversion method. Both experiments cover almost the same mid-rapidity interval. For completeness, Table 1 also includes HELIOS-2 which has performed the first search of direct photons.

\subsection{Dileptons}

The three dilepton experiments have reported, during the last year, an excess of dileptons in S-induced interactions over the known sources as measured in pp or $\mathrm{pA}$ collisions after scaling to the S-nucleus case. The enhancement is beautifully illustrated in Fig. 1 which shows the results of HELIOS-3 in central S-W interactions together with those obtained in p-W at $200 \mathrm{GeV} /$ nucleon [12]. 


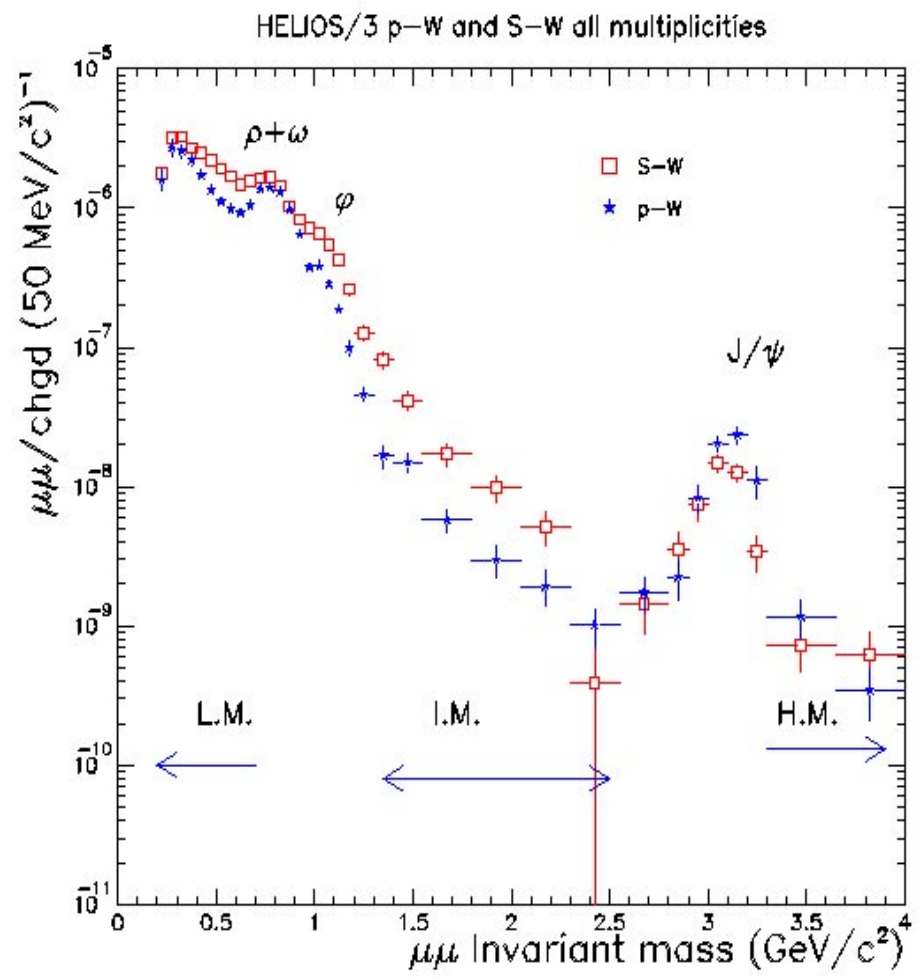

Figure 1. Dimuon invariant mass spectra measured by HELIOS-3 in $p-W$ and in central $\mathrm{S}-\mathrm{W}$ interactions at $200 \mathrm{GeV} /$ nucleon. The data are normalized to the charged particle multiplicity measured in the acceptance of the spectrometer. [12] .

They are presented in the form of muon pairs per charged particle measured in the same rapidity interval. The enhancement covers a very broad mass range including the low-mass continuum $\left(\mathrm{m}=200-600 \mathrm{MeV} / \mathrm{c}^{2}\right)$, the vector mesons $\rho, \omega$ and $\phi$, and the intermediate-mass continuum (above the $\phi$ and below the $J / \psi$ masses). The figure also displays the well known $J / \psi$ suppression, a topic of interest in its own right (see the paper presented by Ramos [13] at this conference).

NA38 has also reported an excess of dimuon pairs in the intermediate mass region. The excess is displayed in Fig. 2 which compares the S-W data with the expected yield from Drell-Yan and semi-leptonic charm decay contributions. These are the main contributions at masses $1.5<\mathrm{m}<2.5 \mathrm{MeV} / \mathrm{c}^{2}$, and they provide a good description of the $\mathrm{p}$ - $\mathrm{W}$ data. For details on the normalization and extrapolation procedures used for the $\mathrm{S}-\mathrm{W}$ data see ref. [14]. A straightforward way to illustrate the excess is shown in Fig. 3 where the p and the $\mathrm{S}$ spectra are overlayed normalizing them in the high mass region $\left(\mathrm{m}>4.2 \mathrm{GeV} / \mathrm{c}^{2}\right)$.

The most dramatic effect is the one observed by the CERES experiment in S-Au collisions as shown in Fig. 4 [15,16]. The results are presented in the form of pair production per event and per charged particle within the acceptance of the spectrometer. The low- 


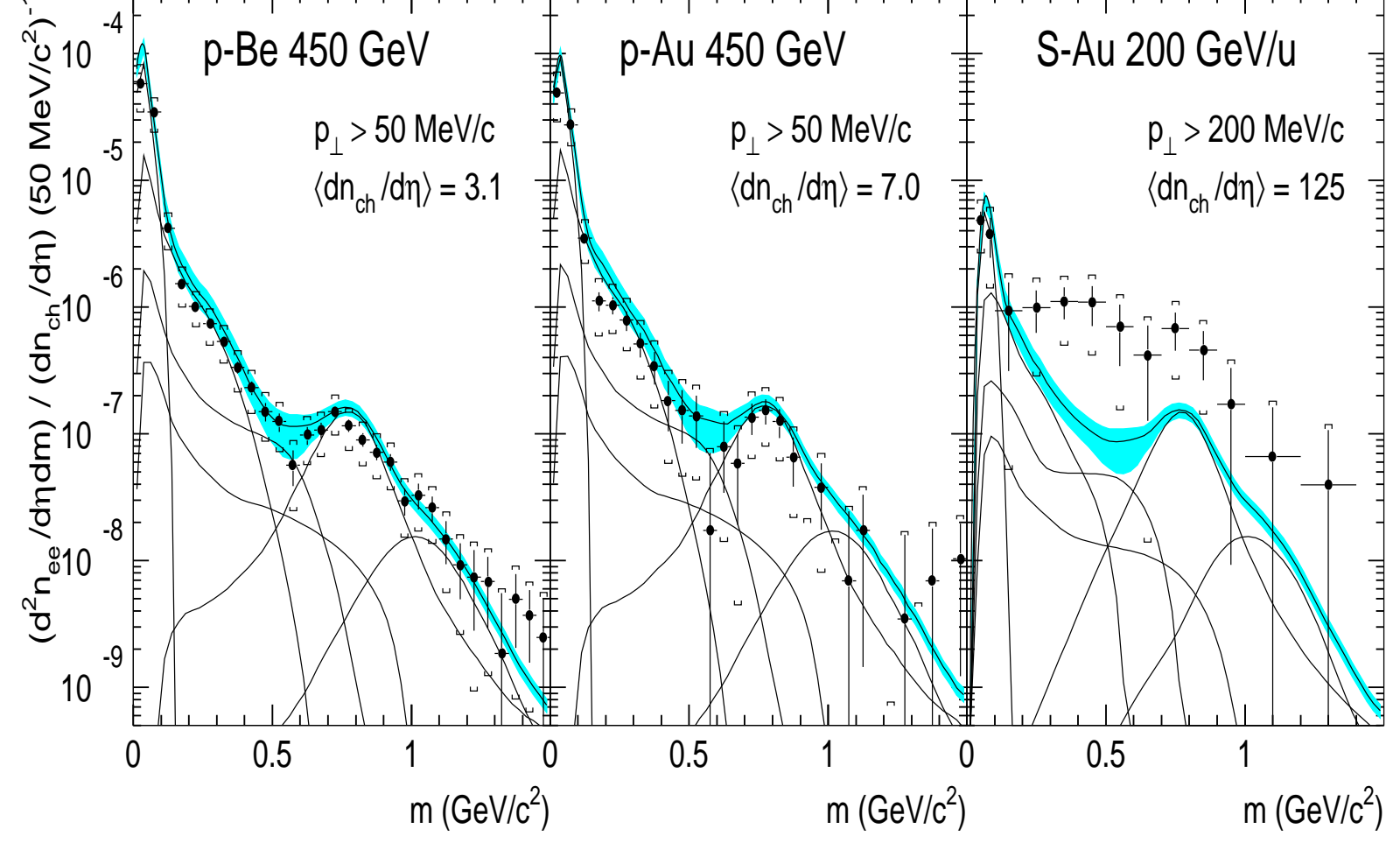

Figure 4. Inclusive $\mathrm{e}^{+} \mathrm{e}^{-}$mass spectra in $450 \mathrm{GeV}$ p-Be and $\mathrm{p}-\mathrm{Au}$ collisions, and in 200 $\mathrm{GeV} /$ nucleon S-Au collisions measured by CERES. The solid lines represent the contributions from the various known hadron decays; the shaded area represent the systematic error on the summed contributions. The data are normalized to give the pair density per charged particle density within the acceptance of the CERES spectrometer $[15,16]$.

consistent with each other and that there is no clear evidence of direct photons within the present level of the systematic errors.

\section{CONSISTENCY AMONG THE VARIOUS EXPERIMENTAL RESULTS}

In this section I shall attempt a more quantitative comparison among the different experimental results, in order to ascertain their consistency. The enhancement factor can be quantified by defining the ratio $[12,15]$ :

$$
\frac{\text { integral of the measured data }}{\text { integral of expected yield from hadronic sources }}
$$

\subsection{Intermediate Mass Range: HELIOS-3 vs NA38}

This is the most straightforward comparison as the two experiments measure dimuons and cover the intermediate mass region where both experiments see an excess of dimuons. The comparison is restricted to the mass region of $\mathrm{m}=1.5-2.5 \mathrm{GeV} / \mathrm{c}^{2}$ where the dominant sources of dimuons are from open charm decays and the Drell-Yan process. 


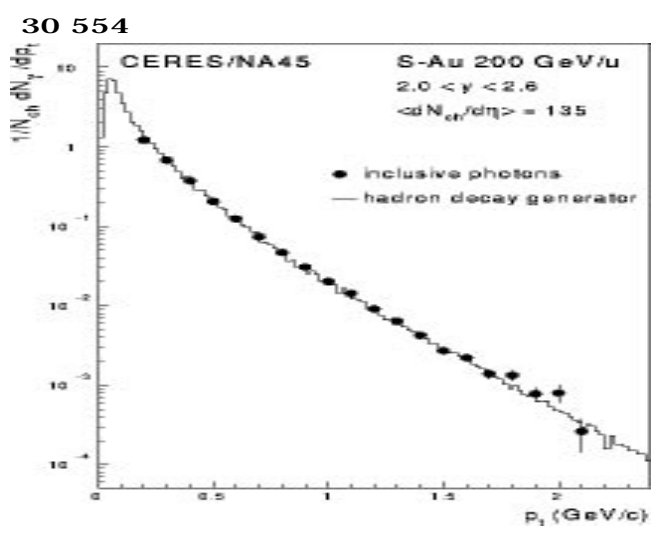

Figure 5. CERES results on inclusive photon $p_{t}$ distribution from central SAu collisions and comparison to predictions from hadron decays [16,19].

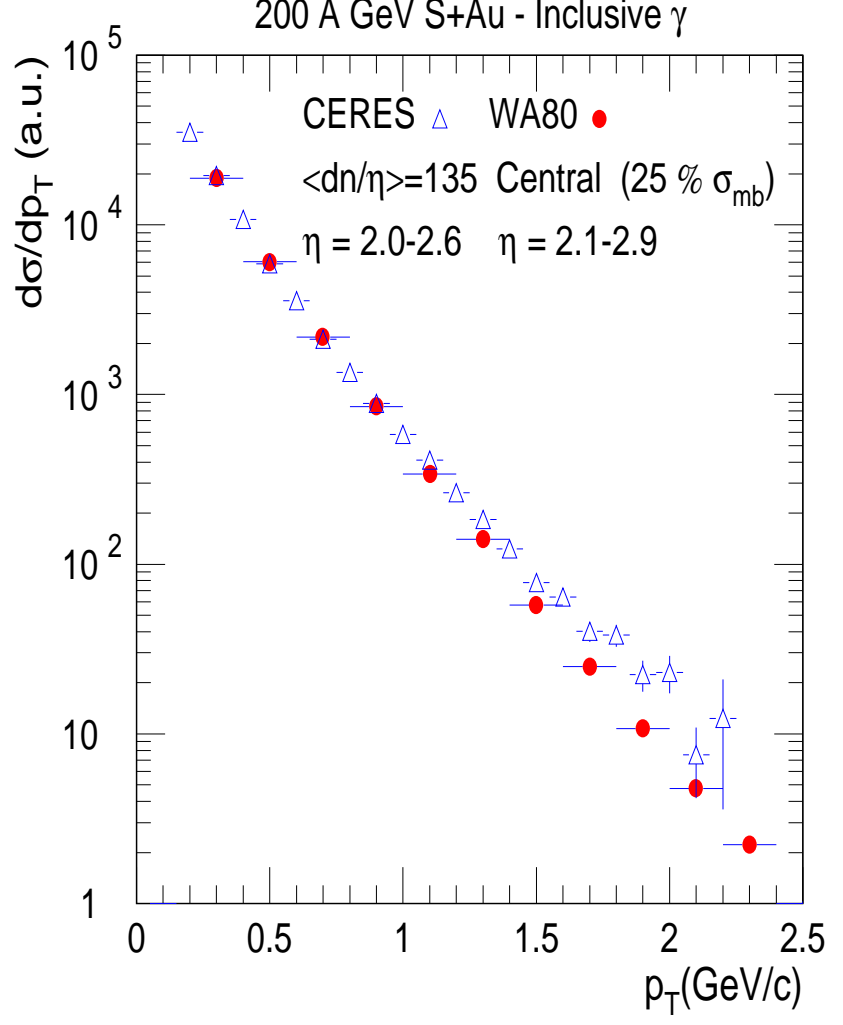

Figure 6. Comparison of the shapes of the photon $p_{t}$ distribution measured by CERES and WA80 central S-Au collisions.

Both experiments have used a similar procedure to evaluate these two contributions. The enhancement factors reported by HELIOS- 3 and NA38 are $2.43 \pm 0.42[12]$ and $1.40 \pm 0.1$ [14], respectively. (Preliminary analysis of the NA38 S-U data taken in 1992 shows an enhancement factor of $1.30 \pm 0.1[24]$ ).

This level of agreement is acceptable if one takes into account that only the statistical errors are quoted, and that there are several differences between the two experiments; in particular they cover different acceptance intervals, and HELIOS-3 has applied a pair acceptance correction whereas NA38 has not.

\subsection{Low-Mass Region: CERES and HELIOS-3}

The low-mass dilepton region has been measured both by CERES and HELIOS-3 in $\mathrm{S}-\mathrm{Au}$ and $\mathrm{S}-\mathrm{W}$ interactions, respectively. The enhancement factor, as defined above, reported by CERES is $5.0 \pm 0.7$ (stat.) \pm 2.0 (syst.) in the mass range of $\mathrm{m}=0.2-1.5 \mathrm{GeV} / \mathrm{c}^{2}$. HELIOS-3 has not yet calculated the expected contributions from hadronic sources in that same mass range. Lacking that, since the $\mathrm{p}$-Au electron pair data are well reproduced by the hadronic sources (see Fig. 4) one can assume with some degree of confidence, that the 
p-W dimuon data will also be accounted for by the known sources; this allows to estimate the enhancement factor as the ratio of the integral of the measured data in $\mathrm{S}-\mathrm{W}$ to the integral of the measured data in $\mathrm{p}-\mathrm{W}$. The HELIOS-3 enhancement factor in the above mass range is then 1.6 , significantly smaller than that of CERES; the discrepancy could be a consequence of the different rapidity coverage of the two experiments, if the dilepton production mechanism has a non-linear (quadratic?) dependence on the event multiplicity. Indeed, CERES covers the mid-rapidity region of $\eta=2.10-2.65$, whereas HELIOS-3 covers the forward region $\eta=3.7-5.5$. Consequently, the average charged-particle rapidity densities accessible by the two experiments, and hence the energy densities, differ by at least a factor of two [25]. This interpretation is supported by preliminary results of HELIOS-3, showing that there is practically no excess at the most forward rapidities $(y>4.4)$ covered by the spectrometer, whereas almost all the excess is at lower rapidities $[26]$.

\subsection{Electron Pair vs. Photon Results of CERES}

Electron pairs and direct photons (or virtual and real photons) are expected to carry the same physical message. It is therefore a very relevant question to ask whether the large enhancement factor observed in electron pairs by CERES is consistent with the lack of signal of photons (or, to be more precise, with a 10\% upper limit for direct photons) measured in the same experiment. In spite of a very extensive theoretical literature on dileptons and photons, there is no quantitative assessment of their relative enhancement factors, as defined above, and I shall provide here only a crude estimate.

The sensitivity to detect any new source of electron pairs or direct photons is determined by the strength of that source with respect to the background of hadronic sources. For the kinematic window covered by the CERES detector, the hadronic background of the photon measurement is larger by more than four orders of magnitude than the corresponding one of the electron-pair measurement (considering only masses $\mathrm{m}>200 \mathrm{MeV} / \mathrm{c}^{2}$ ). This is partially compensated by the larger production cross sections of photons. Any new photon source either partonic or hadronic will have a production cross section proportional to $\alpha \alpha_{s}$ whereas for the electrons the cross section is proportional to $\alpha^{2}$. This gives a factor $\alpha_{s} / \alpha$ in favor of the photons. The resulting level of sensitivity is therefore more than two orders of magnitude lower for photons compared to electrons. In other words, the enhancement factor of 5 observed in the case of electrons should translate into an enhancement factor of a few percent in the photon measurement and this is compatible with the CERES experimental results, within the present level of systematic errors.

The real advantage of the electron pairs over the photons, comes from the additional parameter characterizing the virtual photon, namely its mass. The mass cut of $200 \mathrm{MeV} / \mathrm{c}^{2}$ allows to eliminate the major hadronic source of electron pairs, the $\pi^{\circ}$ Dalitz decays. Without it, the hadronic background would only be two orders of magnitude lower compared to that of the photons, thus bringing the sensitivity of the two probes to approximately the same level.

One should keep in mind that the argument presented above is valid for the integral spectrum. The picture may change if the photon $\mathrm{p}_{t}$ spectra of the new source is different from that of the hadronic sources, allowing to set a $\mathrm{p}_{t}$ cut such that the level of sensitivity becomes much larger. Furthermore, a quadratic dependence of the new source with the 


\section{DISCUSSION AND OUTLOOK}

Three experiments, CERES, HELIOS-3 and NA38 see an enhanced production of dileptons in the low- and the intermediate-mass continuum in S-induced interactions. Although the magnitude of the excess varies from one experiment to another, there is a reasonable degree of consistency among them considering their different kinematic windows. This is an interesting result which is triggering a considerable theoretical activity and which also calls for more experimental information to assess the origin of the excess.

On the theoretical front, it is too early to know whether it will be possible to explain the excess by conventional approaches or whether it will be necessary to modify some of the known processes in the dense matter or to invoke new ones. The attention is mainly focussed on the electron data which are more accessible to calculations using standard hydrodynamical models, since they are measured at mid-rapidity. The characteristic features of the low-mass dilepton excess - its onset at a mass $m_{\ell \ell} \sim 2 m_{\pi}$, its extension to the low-mass region below and around the $\rho$-meson, and the possibility of a quadratic dependence with multiplicity- suggest that the excess is due to the $\pi^{+} \pi^{-}$annihilation into $\ell^{+} \ell^{-}$. This would then be the first indication of radiation emitted from the dense hadronic matter formed in relativistic heavy-ion collisions. As discussed in the introduction, the process is dominated by the electromagnetic form factor of the pion which produces a pronounced peak at the $\rho$-meson mass. However, since the width of the $\rho$-meson is very large, the folding of the cross section with the pion occupation probability in momentum space can give rise to an enhanced yield at lower masses [3], making the $\rho$-peak less pronounced and producing a behaviour similar to what is observed. The pion-annihilation hypothesis is being pursued in a standard hydrodynamical scenario with and without invoking a phase transition to a QGP. Other approaches are also being considered, like for instance a decrease of the $\rho$-meson mass in dense hadronic matter which has been advocated as a precursor of chiral symmetry restoration $[8,9]$.

On the experimental front there are several open questions. Among them, a key question is the multiplicity dependence of the observed excess. The suggestion of a strong dependence derived in the previous section by comparing CERES and HELIOS-3 results is not yet corroborated by a direct measurement. There are no results from CERES on this topic. Masera reported at this conference that the low-mass excess of HELIOS-3 is practically independent of the multiplicity. However, this result should be viewed with caution since the different multiplicity bins are largely overlapping and the range is very limited [12]. NA38 sees a small increase over the linear behavior (definitely not quadratic) of the intermediate mass excess as a function of $\mathrm{E}_{t}$ [24].

Another interesting question concerns the excess below and above the vector mesons $\rho, \omega$ and $\phi$. The HELIOS-3 excess ( $\mathrm{S}-\mathrm{W}$ minus $\mathrm{p}-\mathrm{W}$ yields) plotted as a function of the invariant mass or the transverse mass of the dimuon, can be fitted by a single exponential shape below and above the vector mesons [12]. This behaviour suggests a common origin of the excess in the low- and the intermediate-mass regions, but again the error bars are very large. The results of NA38 point into a different direction. The excess at intermediate masses has a shape very similar to that of the open charm contribution and 
in fact doubling the latter nicely accounts for the excess, suggesting that an enhancement of charm production could be an explanation of the observed effect. One would then need a different explanation for the excess at low masses since charm production has a negligible contribution there.

We also need more precise data on the photons and on their multiplicity dependence for a more meaningful consistency check of the dilepton and the photon results, as discussed in the previous section. The experiments performed so far have been limited by the systematic errors which are now of the order of $10 \%$, and it is therefore clear that great efforts should be made to reduce them. This is not an easy task and the progress so far has been relatively slow. Nevertheless, an improvement of the level of sensitivity to any new source by a factor of 2-3 seems within reach [27].

The new round of experiments at the CERN SPS with Pb beams of $160 \mathrm{GeV} / \mathrm{nucleon}$ which has just started should provide all this information. In addition it will allow to study the evolution of the excess under the better conditions offered by the $\mathrm{Pb}$ beam: the interaction volume increases to the largest value attainable in the laboratory, the multiplicity range also increases by approximately a factor of three and the energy density is somewhat larger.

I am therefore looking forward to the next Quark Matter conference where new results should be available and where we can also expect some more insight from the ongoing theoretical effort.

Acknowledgements: I would like to thank T. Awes, L. Kluberg, G. London, C. Lourenco and M. Masera for discussions on their data and for making their results available for this review. I am specially grateful to G. Brown, K. Redlich, V. Ruuskanen, H. Satz, E.V. Shuryak and D. Srivastava for many discussions about the results and possible interpretations.

\section{REFERENCES}

1. E.V. Shuryak, Phys. Lett. B78, 150 (1978), Sov. J. Nucl. Phys. 28, 408 (1978).

2. K. Kajantie, J. Kapusta, L. McLerran and A. Mekjian, Phys. Rev. D34, 2746 (1986).

3. J. Cleymans, K. Redlich and H. Satz, Z. Phys. C52, 517 (1991).

4. P. Koch, Z. Phys. C57, 283 (1993).

5. Note however, that according to lattice calculations, at $\mathrm{T}>\mathrm{T}_{c}$ the quark mass becomes essentially equal to the pion mass, so that the threshold is remarkably close to $2 \mathrm{~m}_{\pi}$ (E.V. Shuryak, private communication).

6. P.V. Ruuskanen, Nucl. Phys. A544, 169c (1992).

7. J. Cleymans, V.V. Golovizinin and K. Redlich, Z. Phys. C59, 495 (1993).

8. R. Pisarski, Phys. Lett. B110, 155 (1982).

9. G.E. Brown and M. Rho, Phys. Rev. Lett. 66, 2720 (1991).

10. U. Heinz and K.S. Lee, Phys. Lett. B259, 162 (1991).

11. A. Shor, Phys. Rev. Lett. 54, 1122 (1985).

12. M. Masera for the HELIOS-3 Collaboration, these Proceedings.

13. S. Ramos for the NA38 Collaboration, these Proceedings.

14. C. Lourenco, for the NA38 Collaboration, Proc. of the 5th Conference on Intersections 
between Particle and Nulear Physics, St. Petersburg, FL (1994).

15. G. Agakichiev et al., the CERES Collaboration, submitted to Phys. Rev. Lett. and preprint CERN-PPE/95-26.

16. P. Wurm for the CERES Collaboration, these Proceedings.

17. T. Akesson et. al., HELIOS-1 Collaboration, Z. Phys. C to be published.

18. T. Akesson et. al., Phys. Lett. 152B, 411 (1985) and 192B, 463 (1987).

19. D. Irmscher for the CERES Collaboration. (Quark Matter 93, Proc. Int. Conf. on Ultra-Relativistic Nucleus-Nucleus Collisions, Borlänge, Sweden 1993), Nucl. Phys. A566,347c (1994),

20. T. Akesson et al., the HELIOS-2 Collaboration, Z. Phys. C46, 369 (1990).

21. R. Santo et al., the WA80 Collaboration, (Quark Matter 93, Proc. Int. Conf. on UltraRelativistic Nucleus-Nucleus Collisions, Borlänge, Sweden 1993), Nucl. Phys. A566, 61c (1994).

22. R. Albrecht et. al., Z. Phys. C51, 1 (1991).

23. T. Awes for the WA80 Collaboration, these Proceedings.

24. C. Lourenco, NA38 Collaboration, private communication.

25. T. Akesson et. al., HELIOS-2 Collaboration, Nucl. Phys. B353, 1 (1991).

26. G. London, Proceedings of the Workshop on Dilepton Production In Relativistic Heavy ion Collisions, March 2-4, 1994, GSI, Darmstadt, Germany.

27. H. Kraner et. al, CERES Collaboration, Proposal to the SPSLC, CERN SPSLC/941/P280. 